\title{
An integer linear programming approach to find trend-robust run orders of experimental designs
}

\author{
Mr. José Núñez Ares*
}

Dr. Peter Goos ${ }^{\dagger}$

October 10, 2017

\begin{abstract}
When a multi-factor experiment is carried out over a period of time, the responses may depend on a time trend. Unless the tests of the experiment are conducted in a proper order, the time trend has a negative impact on the precision of the estimates of the main effects, the interaction effects and the quadratic effects. A proper run order, called a trend-robust run order, minimizes the confounding between the effects' contrast vectors and the time trend's linear, quadratic and cubic components. Finding a trend-robust run order is essentially a permutation problem. We develop a multistage approach based on integer programming to find a trend-robust run order for any given design. The multistage nature of our algorithm allows us to prioritize the trend robustness of the main-effect estimates. In the literature, most of the methods used are tailored to specific designs, and are not applicable to an arbitrary design. Additionally, little attention has been paid to trend-robust run orders of response surface designs, such as central composite designs, Box-Behnken designs and definitive screening designs. Our algorithm succeeds in identifying trend-robust run orders for arbitrary factorial designs and response surface designs with two up to six factors.
\end{abstract}

Keywords: factorial designs, mixed integer program, multistage approach, orthogonality, response surface designs, trend robustness

\footnotetext{
${ }^{*}$ Doctoral student. Department of Biosystems, KU Leuven, Belgium (jose.nunezares@kuleuven.be)

${ }^{\dagger}$ Professor. Department of Biosystems, KU Leuven, Belgium and Department of Engineering Management, University of Antwerp, Belgium (peter.goos@kuleuven.be)
} 


\section{Time trends and optimal design of experiments}

In this article, we consider multi-factor experiments where the response depends on a polynomial time trend. This may be due to, for example, a learning curve of the person in charge of the experiment, or aging or heating up of the equipment. Cox (1951) presents a single-factor experiment where different treatments are applied to wool, the behaviour of which changes over time. Four other examples of experiments involving time trends are discussed in Joiner and Campbell (1976). Kreutz and Timmer (2009) review experimental design within systems biology and point out that often the cell cycle or circadian processes can cause a systematic trend. Dhanasekaran and Karunanithi (2012) use a linear trend-free Box-Behnken design to investivate the gas holdup in air-water systems. Finally, Villarreal-Marroqun et al. (2017) present an experiment on injection molding, a process for mass-producing plastic components, stressing the importance of having a trend-robust design. In the context of designed experiments, the time trend is usually modeled by means of a low-order polynomial, this is, the time enters the model as a covariate. It is generally assumed that there are no interaction effects between the time trend and the experimental factors.

Consider a face-centered central composite design with two factors and one center point. Table 1 depicts this design in standard order along with a linear time trend, which has been centered around 0 . This design allows the modeling of a second-order relationship between the response and the experimental factors. The estimation of the coefficients of the corresponding second-order model should, ideally, be independent of the time trend. In other words, the coefficients should be estimated as if the trend is absent. This is achieved if the main effects and the second-order effects are orthogonal to the time trend. We then say that the design is trend-robust, trend-free or trend-resistant. Using the standard run order of the design in Table 1, none of the model effects is orthogonal to the linear time trend. For example, the dot product between the main effect contrast vector, $\mathbf{X}_{\mathbf{1}}$, and the linear time trend equals

$$
(-1) \cdot(-4)+(-1) \cdot(-3)+1 \cdot(-2)+1 \cdot(-1)+(-1) \cdot 0+1 \cdot 1+0 \cdot 2+0 \cdot 3+0 \cdot 4=5
$$

Because the dot product differs substantially from zero, the vector $\mathbf{X}_{\mathbf{1}}$ and the linear time trend are far from being orthogonal, forming an angle of, approximately, $75^{\circ}$. Table 2 shows a trend-robust run order for the central composite design considered in which the contrast vectors of all main effects and second-order effects are orthogonal to the linear time trend.

This paper addresses the problem of finding a run order for a given design such that the 


\begin{tabular}{|c|r|r|r|r|r|r|r|r|r|}
\hline Run & $\mathbf{1}$ & $\mathbf{2}$ & $\mathbf{3}$ & $\mathbf{4}$ & $\mathbf{5}$ & $\mathbf{6}$ & $\mathbf{7}$ & $\mathbf{8}$ & $\mathbf{9}$ \\
\hline $\mathbf{X}_{\mathbf{1}}$ & -1 & -1 & 1 & 1 & -1 & 1 & 0 & 0 & 0 \\
\hline $\mathbf{X}_{\mathbf{2}}$ & -1 & 1 & -1 & 1 & 0 & 0 & -1 & 1 & 0 \\
\hline Time & -4 & -3 & -2 & -1 & 0 & 1 & 2 & 3 & 4 \\
\hline
\end{tabular}

Table 1: Face-centered central composite design with two factors and one center point in standard order and linear time trend

\begin{tabular}{|c|r|r|r|r|r|r|r|r|r|}
\hline Run & \multicolumn{1}{c|}{$\mathbf{1}$} & \multicolumn{1}{c|}{$\mathbf{2}$} & \multicolumn{1}{c|}{$\mathbf{1}$} & \multicolumn{1}{c|}{$\mathbf{5}$} & $\mathbf{6}$ & \multicolumn{1}{|c|}{} & $\mathbf{8}$ & $\mathbf{9}$ \\
\hline $\mathbf{X}_{\mathbf{1}}$ & -1 & 1 & 0 & 1 & 0 & -1 & 0 & -1 & 1 \\
\hline $\mathbf{X}_{\mathbf{2}}$ & 0 & -1 & 1 & 1 & 0 & -1 & -1 & 1 & 0 \\
\hline Time & -4 & -3 & -2 & -1 & 0 & 1 & 2 & 3 & 4 \\
\hline
\end{tabular}

Table 2: Trend-robust run order of the face-centered central composite design with two factors and one center point

model effects are orthogonal to linear, quadratic and cubic time trends. In essence, this is a permutation problem. We tackle the problem using mathematical programming techniques, more specifically using integer linear programming.

The literature on trend-robust run orders can be classified in three groups, depending on the solution method used. The first group consists of papers which use algebraic theory and/or foldover techniques. $\operatorname{Cox}(1951)$ was the first to study systematic run orders for an experiment with one qualitative 2-level factor. Box and Hay (1953) study a biological experiment with one quantitative factor, a quadratic regression model in that factor and a time trend which is approximated by a cubic polynomial. They use orthogonal polynomials to code the time trend. Daniel and Wilcoxon (1966) present some results for 2-level full factorial and fractional factorial experiments. Their results are extended by Cheng and Jacroux (1988), who considered 2-level factorial experiments with interactions. John (1990) constructs 2-level and 3-level factorial designs which are robust against linear and quadratic time trends. He considers main effects and interaction effects and uses a foldover technique to find trend-robust run orders. Bailey et al. (1992) present trend-robust run orders for 2level, multi-level and mixed-level factorial designs. They use a generalized foldover method.

The second group is composed of papers that use a point-exchange algorithm to construct D-optimal designs for general models. Atkinson and Donev (1996) present an algorithm that takes into account robustness against linear and quadratic trends. Tack and Vandebroek (2001) build on this article, adding a cost associated with the change of factor levels between consecutive runs. Finally, Tack and Vandebroek (2002) further develop the algorithm by incorporating fixed or random block effects.

The final group includes papers which use integer programming techniques. Our work 
belongs to this category. Hinkelmann and Jo (1998) consider a general Box-Behnken design and propose a system of integer equations which produces a linear trend-free run order. While the previous papers deal with completely randomized designs, Carrano et al. (2006) study the trend robustness of split-plot designs for an experiment with wood performed on abrasive machining. A disadvantage of the approaches of Hinkelmann and Jo (1998) and Carrano et al. (2006) is that they do not provide any run order unless a trend-robust run order exists in which the factor effects and the polynomial time trends are orthogonal.

Recently, integer programming techniques have been used on multiple occasions in the context of optimal design of experiments. Capehart et al. (2011) uses integer programming to design regular fractional factorial split-plot experiments, while Sartono et al. (2015b) use integer programming to block given regular and nonregular orthogonal designs. Capehart et al. (2012) and Sartono et al. (2015a) use integer programming to construct blocked fractional factorial split-plot designs and general orthogonal fractional factorial split-plot designs, respectively.

The contributions of this paper are threefold. First, we present an exact procedure based on integer programming to obtain trend-robust run orders of any given design. Our solution method produces a trend-robust design if such a design exists and if it can be found within the specified computation time limit. Otherwise, it produces a nearly trend-robust design. This is a qualitative improvement over the fold-over techniques and the approach of Hinkelmann and Jo (1998), because these techniques fail to produce a run order unless a trend-robust one exists. Unlike the approach of Atkinson and Donev (1996), our solution method is sequential in nature, and this allows us to prioritize the robustness of main effects and second-order effects against linear, quadratic and cubic time trends. So, our method prioritizes the robustness of the most important effects against the most important kinds of time trends. Second, we apply our method to different response surface designs that have not been studied before in the context of trend robustness, such as (small) central composite designs, Box-Behnken designs with more than three factors and definitive screening designs. We also improve some previously published results. Finally, we introduce a new measure for the trend robustness of designs.

The remainder of this paper is organized as follows. Section 2 describes the different types of response surface designs we study and introduces our notation. In Section 3, we define our problem and discuss its most important features. In Section 4 , we introduce our model and our algorithm, and briefly discuss the complexity. Next, we present our results in 
Section 5. Finally, in Section 6, we draw conclusions, discuss possible directions for future research and describe three related problems that can be solved by means of our approach.

\section{Preliminaries}

In this section, we briefly describe the design types that we have used in our work and introduce the notation used for our statistical model.

\subsection{Factorial designs and response surface designs}

Response surface methodology aims to estimate a quadratic regression model that (locally) approximates the relation between the response and the experimental factors. We have studied the trend robustness of factorial designs with two to five factors and three, four or five levels per factor. While these designs can be used to estimate curvature, their size grows exponentially with the number of factors.

As an alternative, Box and Wilson (1951) introduced the central composite designs to estimate quadratic models. These designs consist of a 2-level factorial design, a set of axial points and some center points. Hartley (1959) presented the small central composite designs, which use a 2-level fractional factorial design of resolution III. For both designs, there are several options for the axial points. In the present paper, we focus on so-called face-centered central composite designs, because the integer nature of their factor levels makes these designs more suitable for use in the presence of time trends than rotatable or spherical central composite designs. Box and Behnken (1960) presented another design which is also suitable for fitting a quadratic model. These designs combine a 2-level (fractional) factorial design with an incomplete block design and a set of center points. Jones and Nachtsheim (2011) presented the definitive screening designs as a cost-efficient alternative with which, for large numbers of factors, any 3-factor full quadratic model can be estimated. We have studied the trend-robustness of central composite designs and Box-Behnken designs with 2 up to 5 factors, and small central composite designs and definitive screening designs with 2 up to 6 factors.

Central composite, Box-Behnken and definitive screening designs can be extended by adding center runs. A definitive screening design can be expanded in another fashion as 
well. For example, the standard designs for 3 and 4 factors have 9 and 13 runs, respectively. If we consider the first 3 columns (corresponding to the first three factors) of the 4-factor definitive screening design, we obtain a 13-run definitive screening design for a model with 3 factors. We also test whether these extensions lead to an increase in the robustness against polynomial time trends.

\subsection{Second-order statistical model}

We assume that the statistical model of interest includes the main effects, interaction effects and quadratic effects of the $m$ quantitative factors and a polynomial time trend up to the cubic degree. In matrix notation, and considering $n$ runs, the model is given by

$$
\mathbf{Y}=\mathbf{X} \boldsymbol{\beta}+\mathbf{Z} \gamma+\epsilon
$$

where

$$
\mathbf{X}=\left[\begin{array}{c:ccc:cccccc}
1 & X_{11} & \ldots & X_{m 1} & X_{11} X_{21} & \ldots & X_{m-1,1} X_{m 1} & X_{11}^{2} & \ldots & X_{m 1}^{2} \\
1 & X_{12} & \ldots & X_{m 2} & X_{12} X_{22} & \ldots & X_{m-1,2} X_{m 2} & X_{12}^{2} & \ldots & X_{m 2}^{2} \\
\vdots & \vdots & \ddots & \vdots & \vdots & \ddots & \vdots & \vdots & \ddots & \vdots \\
1 & X_{1 n} & \ldots & X_{m n} & X_{1 n} X_{2 n} & \ldots & X_{m-1, n} X_{m n} & X_{1 n}^{2} & \ldots & X_{m n}^{2}
\end{array}\right]
$$

and

$$
\mathbf{Z}=\left[\begin{array}{c:c:c}
Z_{11} & Z_{21} & Z_{31} \\
Z_{12} & Z_{22} & Z_{32} \\
\vdots & \vdots & \vdots \\
Z_{1 n} & Z_{2 n} & Z_{3 n}
\end{array}\right]
$$

and $\boldsymbol{\epsilon}$ is the vector of random errors. The matrix $\mathbf{X}$ is the model matrix for the experimental factors and $\mathbf{Z}$ is the model matrix for the time trend, where $Z_{d t}$ is the time trend component of degree $d$ for time $t$. Finally, $\boldsymbol{\beta}$ is the vector containing the intercept, main effects and second-order effects and $\gamma$ is the parameter vector containing the linear, quadratic and cubic time trend effects. We consider the following partitions of the matrices $\mathbf{X}$ and $\mathbf{Z}$ :

$$
\mathbf{X}=\left[\begin{array}{lll}
\mathbf{1} & \mathbf{X}_{M E} & \mathbf{X}_{S O E}
\end{array}\right]
$$


and

$$
\mathbf{Z}=\left[\begin{array}{lll}
\mathbf{Z}_{L} & \mathbf{Z}_{Q} & \mathbf{Z}_{C}
\end{array}\right]
$$

where $\mathbf{1}$ is the column of ones in the model matrix $\mathbf{X}, \mathbf{X}_{M E}$ is the part of the model matrix $\mathbf{X}$ corresponding to the main effects and $\mathbf{X}_{S O E}$ is the part corresponding to the second-order effects. Likewise, $\mathbf{Z}_{L}$ corresponds to the linear component of the time trend, $\mathbf{Z}_{Q}$ to the quadratic component and $\mathbf{Z}_{C}$ to the cubic time trend component.

\section{Problem description}

The input data of our problem consists of given response surface designs whose runs are arranged in the standard order. Our goal is to re-order the runs such that the resulting designs are robust against a linear, quadratic and cubic time trend. Assuming a suitable coding is used for the time trend components, this is achieved when all entries of the matrix product $\mathbf{X}^{T} \mathbf{Z}$ are zero.

\subsection{Coding of the polynomial time trends}

The polynomial time trends can be coded in different ways. An intuitive way is to use consecutive integer numbers for the linear trend component, square these numbers for the quadratic time trend component and use their third power for the cubic component. A mathematically more elegant option is to use orthogonal polynomials, which is the coding we have chosen.

Orthogonal polynomials have originally been introduced to simplify the calculations necessary to fit statistical models. Fisher and Yates (1963), in their Table XXIII, show the orthogonal polynomial coding for time trends up to degree five for different numbers of runs. To automate the generation of the coding, we follow Van der Reyden (1957). This implies that the time trend components $Z_{1 t}, Z_{2 t}$ and $Z_{3 t}$ of degree 1,2 and 3 for time $t$ are calculated as

$$
\begin{aligned}
& Z_{1 t}=t-1-\frac{n-1}{2} \\
& Z_{2 t}=(t-1)^{2}-\frac{1}{12}\left(n^{2}-1\right)
\end{aligned}
$$




\begin{tabular}{|c|r|r|r|r|r|r|r|r|r|r|c|}
\hline $\mathbf{t}$ & $\mathbf{1}$ & $\mathbf{2}$ & $\mathbf{3}$ & $\mathbf{4}$ & $\mathbf{5}$ & $\mathbf{6}$ & $\mathbf{7}$ & $\mathbf{8}$ & $\mathbf{9}$ & $\mathbf{1 0}$ & $\mathbf{1 1}$ \\
\hline$Z_{1 t}$ & -5 & -4 & -3 & -2 & -1 & 0 & 1 & 2 & 3 & 4 & 5 \\
\hline$Z_{2 t}$ & 15 & 6 & -1 & -6 & -9 & -10 & -9 & -6 & -1 & 6 & 15 \\
\hline$Z_{3 t}$ & -30 & 6 & 22 & 23 & 14 & 0 & -14 & -23 & -22 & -6 & 30 \\
\hline
\end{tabular}

Table 3: Orthogonal coding of a cubic time trend for an experiment with 11 runs

and

$$
Z_{3 t}=(t-1)^{3}-\frac{1}{20}\left(3 n^{2}-7\right)(t-1)
$$

Whenever these expressions yield fractional results, we multiply these results by an appropriate integer to obtain integer values for $Z_{1 t}, Z_{2 t}$ and $Z_{3 t}$.

Table 3 shows the orthogonal coding of a cubic time trend for an experiment with 11 runs. The rows of the table show the entries $Z_{1 t}, Z_{2 t}$ and $Z_{3 t}$ of the vectors $\mathbf{Z}_{\mathbf{L}}, \mathbf{Z}_{\mathbf{Q}}$ and $\mathbf{Z}_{\mathbf{C}}$. The entries of each of the vectors sum to zero, and the vectors are orthogonal to each other. This coding has the major advantage that it decomposes the time trend in independent components. This allows us, for example, to make independent statements about the robustness of run orders of experimental designs against linear, quadratic and cubic time trend components.

\subsection{Measure of the trend robustness}

Let $\mathbf{X}_{k}$ be the $k$ th column of the model matrix $\mathbf{X}$ for the experimental factors and $\mathbf{Z}_{d}$ be any column of the model matrix $\mathbf{Z}$ for the time trend. To measure the (lack of) orthogonality between the model effect corresponding to $\mathbf{X}_{k}$ and the time trend component corresponding to $\mathbf{Z}_{d}$, we use the absolute value of the correlation between the column vectors $\mathbf{X}_{k}$ and $\mathbf{Z}_{d}$,

$$
\left|\cos \alpha_{k d}\right|=\frac{\left|\left\langle\mathbf{X}_{k}, \mathbf{Z}_{d}\right\rangle\right|}{\left\|\mathbf{X}_{k}\right\|\left\|\mathbf{Z}_{d}\right\|}
$$

where $\alpha$ is the angle between the vectors.

The absolute correlation (also called cosine similarity) is one of the most commonly used measures of proximity between two vectors in data mining (see Section 2.4 in Tan et al. (2014), for example). The absolute correlation takes values between 0 and 1 . A zero value indicates perfect orthogonality between two vectors, this is, an angle of $90^{\circ}$, whereas a value of one indicates linear dependence or perfect collinearity, this is, an angle of $0^{\circ}$. Using this 
measure, we can evaluate the quality of a run order whenever a completely trend-robust run order cannot be achieved. For example, if $\left|\cos \alpha_{k d}\right| \leq 0.01$, then the vectors $\mathbf{X}_{k}$ and $\mathbf{Z}_{d}$ form an angle $\alpha_{k d}$ within the interval $\left[89.43^{\circ}, 90.57^{\circ}\right]$, indicating that they are nearly orthogonal. When $\left|\cos \alpha_{k d}\right| \leq 0.1$, then $\alpha_{k d} \in\left[84.26^{\circ}, 95.74^{\circ}\right]$, and, when $\left|\cos \alpha_{k d}\right| \leq 0.25$, $\alpha_{k d} \in\left[75.52^{\circ}, 104.48^{\circ}\right]$.

\section{A multistage approach to obtain optimal run orders}

In this section, we explain how our problem can be modeled using a mixed integer linear program. First, we present the objective function and a one-step optimization problem that attempts to solve the problem at once. We then explain that this approach is not sophisticated enough to cope with the particularities of our problem. This is because it fails to prioritize the robustness of the most important factor effects against the most important time trend components. Finally, we present a multistage approach to overcome this issue.

\subsection{Objective function}

Let $\mathbf{X}$ be the given initial $n \times m$ model matrix and let $\mathbf{X}^{*}$ be another $n \times m$ matrix, the rows of which are a permutation of the rows of $\mathbf{X}$. Then, both matrices are related through an $n \times n$ permutation matrix $\mathbf{P}$ where the $(i, j)$ th element of which equals 1 if the $j$ th run in $\mathbf{X}$ is performed at time $i$, and zero otherwise. In matrix form, $\mathbf{X}^{*}=\mathbf{P X}$. As before, let $\mathbf{Z}$ be the $n \times q$ design model matrix for the time trends, where $q$ is the number of time trend components.

To quantify the extent to which the run order given by $\mathbf{X}^{*}$ is trend-robust, we consider an optimality criterion based on the vector 1 - norm applied to matrices, this is, the sum of the absolute values of the entries of the matrix. As an example, consider the problem of finding a run order of a given design which ensures that the main effects are orthogonal to the linear and quadratic time trend components. In that case, our interest is in $\mathbf{X}=\mathbf{X}_{\mathbf{M E}}$ and $\mathbf{Z}=\left[\begin{array}{ll}\mathbf{Z}_{L} & \mathbf{Z}_{Q}\end{array}\right]$, and we want all entries of the matrix $\mathbf{A}=\left(\mathbf{P X}_{\mathbf{M E}}\right)^{T}\left[\begin{array}{ll}\mathbf{Z}_{L} & \mathbf{Z}_{Q}\end{array}\right]$ to equal zero. To achieve this goal, we minimize the sum of the absolute values of the entries of $\mathbf{A}$. This is equivalent to minimizing $\sum_{k=1}^{m} \sum_{l=1}^{2}\left|A_{k l}\right|$, where $A_{k l}$ is the entry of $\mathbf{A}$ in row $k$ and column $l$. This sum is the $1-$ norm of $\mathbf{A}$ and it is denoted as $\|\mathbf{A}\|_{1}$. The minimum of $\|\mathbf{A}\|_{1}$ is zero if complete orthogonality with respect to the time trend can be achieved. 


\subsection{One-step optimization}

Let $\Pi_{n}$ be the set of permutation matrices $\mathbf{P}$ of size $n \times n$. To find the best possible run order in terms of trend robustness, we need to solve the following optimization problem:

$$
\min _{\mathbf{P} \in \Pi_{n}}\left\|(\mathbf{P X})^{T} \mathbf{Z}\right\|_{1}
$$

This permutation problem can also be expressed as an integer programming problem. As a matter of fact, using the binary variables

$$
\pi_{i j}=\left\{\begin{array}{lc}
1, & \text { if run } i \text { is performed at time } j \\
0, & \text { otherwise }
\end{array}\right.
$$

we can express the $(j, k)$ th entry of $\mathbf{X}^{*}=\mathbf{P X}$ as

$$
X_{j k}^{*}=\sum_{i=1}^{n} X_{i k} \pi_{i j}
$$

The $(k, d)$ th element of the matrix product $\mathbf{X}^{* T} \mathbf{Z}$, denoted by $\gamma_{k d}$, is given by

$$
\gamma_{k d}=\sum_{j=1}^{n} X_{j k}^{*} Z_{j d}=\sum_{i=1}^{n} \sum_{j=1}^{n} X_{i k} \pi_{i j} Z_{j d}
$$

The objective function minimizes the sum of all absolute entries of the matrix product $\mathbf{X}^{* T} \mathbf{Z}$ :

$$
\min \sum_{k=1}^{m} \sum_{t=1}^{p}\left|\gamma_{k d}\right|
$$

The absolute values in this expression can be modeled using extra non-negative continuous variables in the integer program, $\gamma_{k d}^{+}$and $\gamma_{k d}^{-}$, for all $k$ and $d$ values, and by adding the following constraint to the model for each value of $k$ and $d$ :

$$
\gamma_{k d}=\gamma_{k d}^{+}-\gamma_{k d}^{-}
$$

In the event $\gamma_{k d}$ is positive, this constraint ensures that $\gamma_{k d}^{+}=\gamma_{k d}$ and $\gamma_{k d}^{-}=0$. Otherwise, it ensures that $\gamma_{k d}^{-}=-\gamma_{k d}$ and $\gamma_{k d}^{+}=0$. Using the new variables, $\gamma_{k d}^{+}$and $\gamma_{k d}^{-}$, the objective function can be modified to

$$
\min \sum_{k=1}^{m} \sum_{d=1}^{p}\left(\gamma_{k d}^{+}+\gamma_{k d}^{-}\right)
$$

The complete integer program that will output the desired permutation of the rows of 
the matrix $\mathbf{X}$ can therefore be formulated as follows:

$$
\begin{aligned}
& \operatorname{minimize} \sum_{k=1}^{m} \sum_{d=1}^{p}\left(\gamma_{k d}^{+}+\gamma_{k d}^{-}\right) \\
& \text {s. t. } \quad \gamma_{k d}^{+}-\gamma_{k d}^{-}=\sum_{i=1}^{n} \sum_{j=1}^{n} X_{i k} \pi_{i j} Z_{j d} \quad \forall k, d \\
& \sum_{j=1}^{n} \pi_{i j}=1 \quad \forall i \\
& \sum_{i=1}^{n} \pi_{i j}=1 \quad \forall j \\
& \gamma_{k d}^{+}, \gamma_{k d}^{-} \geq 0 \quad \forall k, d \\
& \pi_{i j} \in\{0,1\} \quad \forall i, j
\end{aligned}
$$

The objective function 12 is the 1 -norm of the $m \times p$ matrix product $\mathbf{X}^{* T} \mathbf{Z}$. With constraints (13), we model the absolute value of each entry of $\mathbf{X}^{* T} \mathbf{Z}$. Constraints (14) ensure that each run of $\mathbf{X}$ appears in the optimal run order once and constraints (15) ensure that exactly one run is assigned to each of the $n$ time points. Constraints (14) and (15) are known as assignment constraints. Finally, constraints (16) impose the non-negativity of the variables that model the absolute value and constraints (17) define the permutation variables as binary.

The integer program defined by Equations (12)-(17) is intuitive, but it is not ideal to solve our problem because it does not prioritize the robustness of the most important factor effects, i.e. the orthogonality of the main effects with respect to the most important time trend components. For example, it treats the orthogonality of the main effects and linear time trend component as equally important as the orthogonality of the second-order effects and cubic time trend component. We believe that this is in conflict with the fact that higherorder terms are generally less important than lower-order terms. 


\subsection{Multistage approach}

We aim to prioritize the trend-robustness of the main effects estimates and to attach a larger importance to the linear time trend component than to the quadratic and cubic time trend components. This means that our procedure should pay attention to robustness against the quadratic trend component only if the robustness against the linear time trend component is optimal. This suggests a multistage approach, in which we solve the following optimization problems one by one

1. a) Maximize the orthogonality between the main effects and the linear time trend component;

b) Maximize the orthogonality between the second-order effects and the linear time trend component without worsening the trend robustness achieved so far;

2. a) Maximize the orthogonality between the main effects and the quadratic time trend component without worsening the trend robustness achieved so far;

b) Maximize the orthogonality between the second-order effects and the quadratic time trend component without worsening the trend robustness achieved so far;

3. a) Maximize the orthogonality between the main effects and the cubic time trend component without worsening the trend robustness achieved so far;

b) Maximize the orthogonality between the second-order effects and the cubic time trend component without worsening the trend robustness achieved so far.

In technical terms, the first two steps of our multistage approach can be formulated as follows. Our first priority is to seek a run order for which the main effect estimates are as robust as possible to the linear time trend component. This optimization problem is given by

$$
\min _{\mathbf{P} \in \Pi_{n}}\left\|\left(\mathbf{P} \mathbf{X}_{M E}\right)^{T} \mathbf{Z}_{L}\right\|_{1}
$$

Now, suppose that the optimal objective function value for this problem is $O P T_{1}$. The next stage of the approach seeks a run order for which the second-order effects are as robust as possible to the linear time trend component, while respecting the robustness of the main effects to that component. This is done by solving the following minimization problem: 


$$
\begin{aligned}
& \min _{\mathbf{P} \in \Pi_{n}}\left\|\left(\mathbf{P} \mathbf{X}_{S O E}\right)^{T} \mathbf{Z}_{L}\right\|_{1} \\
& \text { s.t. }\left\|\left(\mathbf{P X}_{M E}\right)^{T} \mathbf{Z}_{L}\right\|_{1} \leq O P T_{1}
\end{aligned}
$$

Our complete multistage algorithm is outlined in pseudocode in Algorithm 1. First, we define the set of factor effects, $S_{E F F E C T S}$, which has two elements, main effects and second-order effects (interactions and quadratic effects). Next, we define the set of time trend components, $S_{\text {TIMETRENDS }}$, which has three elements: linear, quadratic and cubic. We also define a set of constraints $C$, which is empty at the start of the algorithm. The algorithm ends when all combinations of factor effects and time trend components have been optimized, so that, in total, six optimization problems are solved. Note that we can also consider a model with only main effects and then only three optimizations are required. The results we report in Section 5 are obtained by applying this multistage optimization to a model with only main effects and to a full quadratic model.

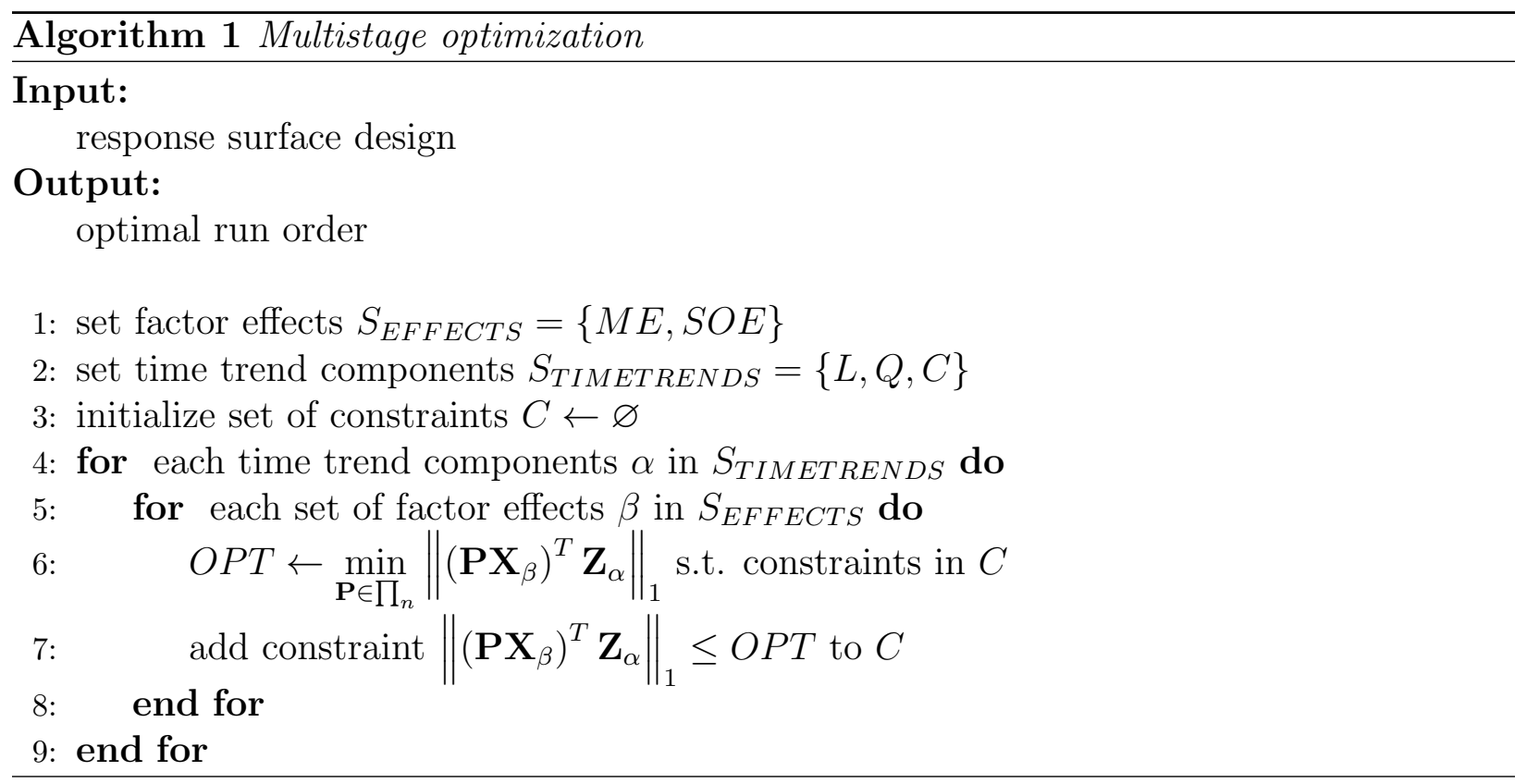

\subsection{Complexity}

Our solution approach is based on the 1-norm minimization. To show that this problem is NP-hard, we show how another NP-hard problem naturally reduces to the 1-norm minimiza- 
tion. NP-hardness is a property introduced by Cook (1971) that defines a class of problems characterized by their intractability. Consider an arbitrary design with model matrix $\mathbf{X}$ and a set of covariates contained in the matrix $\mathbf{Z}$. It is straightforward to see that the partition problem (see p. 223 in Garey and Johnson (1979)) reduces to our integer optimization problem. Given a set of integers, the partition problem looks for two subsets such that the sum of the elements in each subset is equal. This problem is known to be NP-hard, also when those subsets are of equal size. To see the reduction, consider a matrix $\mathbf{X}$ which has only one column containing an equal number of +1 s and -1 s. We include the elements of

the partition set in the column vector $\mathbf{Z}$. Then, if $\min _{\mathbf{P} \in \Pi_{n}}\left\|(\mathbf{P X})^{T} \mathbf{Z}\right\|_{1}$ equals zero, we have found a solution to the partition problem. Therefore, in general, our mixed integer problem is NP-hard, so our optimization algorithm based on integer programming techniques is justified.

\section{Computational results}

\subsection{Choice of software}

Table 4 presents the results of our multistage approach for five different response surface designs, considering a model with main effects, interaction effects and quadratic effects. To search for trend-robust run orders of the five designs, we initially used two commercial integer programming software packages, namely SAS/OR 9.4 and ILOG CPLEX 12.61, set the time limit for the computations to one hour and used a computer with a $2.3 \mathrm{GHz}$ i7-4712HQ processor with $16 \mathrm{~GB}$ of RAM.

Table 4 presents the computation times for both software packages for the following five designs:

1. Box-Behnken design with 3 factors and 3 center points

2. Box-Behnken design with 4 factors and 3 center points

3. Face-centered central composite design (full factorial) with 3 factors and 3 center points

4. Spherical central composite design (full factorial) with 3 factors and 3 center points

5. Definitive screening design with 6 factors

Our results show that, in terms of computing time, CPLEX generally performs better than SAS, which is unable to find a solution within the time limit for some optimization problems. 


\begin{tabular}{|c|c|c|c|c|c|c|c|}
\hline \multirow{2}{*}{ Design } & \multirow{2}{*}{ Software } & \multicolumn{7}{|c|}{ Optimization } \\
\cline { 3 - 8 } & & $\mathbf{1 a}$ & $\mathbf{1 b}$ & $\mathbf{2 a}$ & $\mathbf{2 b}$ & $\mathbf{3 a}$ & $\mathbf{3 b}$ \\
\hline \multirow{2}{*}{$\mathbf{1}$} & SAS & 0.1 & 0.1 & 0.1 & 3.6 & 7.3 & 1.3 \\
\cline { 2 - 8 } & CPLEX & 0.1 & 0.1 & 0.3 & 0.2 & 0.3 & 0.1 \\
\hline \multirow{2}{*}{$\mathbf{2}$} & SAS & 3.3 & 2.8 & 194.3 & $*$ & $*$ & $*$ \\
\cline { 2 - 8 } & CPLEX & 0.5 & 98.6 & 697.7 & 713.6 & $*$ & $*$ \\
\hline \multirow{2}{*}{3} & SAS & 1.4 & 2.1 & 3.0 & 59.7 & 107.1 & 1.6 \\
\cline { 2 - 8 } & CPLEX & 0.1 & 0.1 & 0.1 & 3.2 & 5.2 & 0.2 \\
\hline \multirow{2}{*}{4} & SAS & 4.8 & 191.9 & 215.0 & $*$ & $*$ & $*$ \\
\cline { 2 - 8 } & CPLEX & 0.1 & 0.7 & 6.7 & 1302.7 & 2323.3 & 308.0 \\
\hline \multirow{2}{*}{5} & SAS & 20.1 & 1403.6 & 258.9 & 53.2 & 289.5 & 783.9 \\
\cline { 2 - 8 } & CPLEX & 2.1 & 8.7 & 38.9 & 5.5 & 8.1 & 9.3 \\
\hline
\end{tabular}

Table 4: Computation times (in sec) of SAS and CPLEX for the six optimization problems in our multistage approach

The cases for which SAS failed to produce a solution within the computing time limit are indicated with an asterisk

Because of the relatively poor performance of SAS for these test instances, we used CPLEX for the remainder of our research. Note that we use the default built-in branch-andcut solution scheme of CPLEX 12.61, which provides a bound for the optimal value of the objective function. This is useful when the optimality of the solution provided cannot be established (usually when large designs are considered), because it gives us information on the maximum gap between the current solution and the best possible solution.

\section{$5.2 \quad$ Factorial designs}

We have studied the trend robustness of the $3^{2}, 3^{3}, 3^{4}, 3^{5}, 4^{2}, 4^{3}, 4^{4}, 5^{2}, 5^{3}$ and $5^{4}$ factorial designs. In doing so, we treated all the factors as quantitative factors. In this section, we first present our results for a $3^{3}$ factorial experiment in detail. Next, we summarize our findings for the other factorial designs.

\subsection{1 $\quad 3^{3}$ factorial design}

For the $3^{3}$ factorial design, we have run our algorithm twice. First, we considered a model with main effects only. Next, we considered a full quadratic model. We have therefore obtained two attractive run orders, which we present in Table 5 together with the standard run order and the orthogonal coding of the time trend components up to a cubic degree.

The extent to which the initial run order is trend-robust is quantified in Table 6. The table shows the average and maximum value of the absolute correlation (see Section 3.2) 


\begin{tabular}{|c|c|c|c|c|c|c|c|c|c|c|c|c|}
\hline \multirow[b]{2}{*}{$t$} & \multicolumn{3}{|c|}{$\begin{array}{c}\text { Standard run } \\
\text { order }\end{array}$} & \multicolumn{3}{|c|}{ Run order 1} & \multicolumn{3}{|c|}{ Run order 2} & \multicolumn{3}{|c|}{ Time trend } \\
\hline & $X_{1 t}$ & $X_{2 t}$ & $X_{3 t}$ & $X_{1 t}$ & $X_{2 t}$ & $X_{3 t}$ & $X_{1 t}$ & $X_{2 t}$ & $X_{3 t}$ & $Z_{1 t}$ & $Z_{2 t}$ & $Z_{3 t}$ \\
\hline 1 & -1 & -1 & -1 & 1 & 1 & 1 & -1 & 1 & -1 & -13 & 325 & -130 \\
\hline 2 & -1 & -1 & 0 & -1 & -1 & 0 & -1 & 0 & 1 & -12 & 250 & -70 \\
\hline 3 & -1 & -1 & 1 & 0 & -1 & -1 & 0 & -1 & 0 & -11 & 181 & -22 \\
\hline 4 & -1 & 0 & -1 & -1 & 1 & 0 & 0 & 1 & 1 & -10 & 118 & 15 \\
\hline 5 & -1 & 0 & 0 & -1 & -1 & -1 & 1 & -1 & 0 & -9 & 61 & 42 \\
\hline 6 & -1 & 0 & 1 & 1 & 0 & 0 & -1 & -1 & -1 & -8 & 10 & 60 \\
\hline 7 & -1 & 1 & -1 & 1 & 1 & -1 & 1 & 0 & 0 & -7 & -35 & 70 \\
\hline 8 & -1 & 1 & 0 & 1 & 0 & 1 & 1 & 1 & -1 & -6 & -74 & 73 \\
\hline 9 & -1 & 1 & 1 & 0 & 1 & 1 & 1 & 0 & 1 & -5 & -107 & 70 \\
\hline 10 & 0 & -1 & -1 & -1 & 1 & 1 & 1 & 1 & 0 & -4 & -134 & 62 \\
\hline 11 & 0 & -1 & 0 & 1 & -1 & -1 & 0 & -1 & 1 & -3 & -155 & 50 \\
\hline 12 & 0 & -1 & 1 & 0 & 0 & 0 & 1 & -1 & -1 & -2 & -170 & 35 \\
\hline 13 & 0 & 0 & -1 & 1 & -1 & 0 & 0 & 0 & -1 & -1 & -179 & 18 \\
\hline 14 & 0 & 0 & 0 & -1 & -1 & 1 & 0 & 0 & 0 & 0 & -182 & 0 \\
\hline 15 & 0 & 0 & 1 & -1 & 0 & 0 & 0 & 0 & 1 & 1 & -179 & -18 \\
\hline 16 & 0 & 1 & -1 & -1 & 0 & 1 & -1 & 1 & 1 & 2 & -170 & -35 \\
\hline 17 & 0 & 1 & 0 & -1 & 0 & -1 & 0 & 1 & -1 & 3 & -155 & -50 \\
\hline 18 & 0 & 1 & 1 & 1 & 0 & -1 & -1 & -1 & 0 & 4 & -134 & -62 \\
\hline 19 & 1 & -1 & -1 & 0 & 1 & -1 & -1 & 0 & -1 & 5 & -107 & -70 \\
\hline 20 & 1 & -1 & 0 & 1 & 1 & 0 & -1 & -1 & 1 & 6 & -74 & -73 \\
\hline 21 & 1 & -1 & 1 & 0 & -1 & 0 & -1 & 0 & 0 & 7 & -35 & -70 \\
\hline 22 & 1 & 0 & -1 & 0 & 0 & 1 & 1 & 1 & 1 & 8 & 10 & -60 \\
\hline 23 & 1 & 0 & 0 & 0 & 1 & 0 & -1 & 1 & 0 & 9 & 61 & -42 \\
\hline 24 & 1 & 0 & 1 & 0 & 0 & -1 & 0 & -1 & -1 & 10 & 118 & -15 \\
\hline 25 & 1 & 1 & -1 & 0 & -1 & 1 & 0 & 1 & 0 & 11 & 181 & 22 \\
\hline 26 & 1 & 1 & 0 & 1 & -1 & 1 & 1 & 0 & -1 & 12 & 250 & 70 \\
\hline 27 & 1 & 1 & 1 & -1 & 1 & -1 & 1 & -1 & 1 & 13 & 325 & 130 \\
\hline
\end{tabular}

Table 5: Standard and trend-robust run orders of a $3^{3}$ design with coded time trend

between the model effects (main effects, interaction effects and quadratic effects) and the linear, quadratic and cubic time trend components. For example, the pair of values in the upper left corner of the table, $(0.454,0.943)$, shows the average and maximum value of the absolute correlation between main effects and the linear time trend component. We see that, within the standard run order, the main effects are not at all trend-robust. Surprisingly, the main effects are orthogonal to the quadratic time trend component and the second-order effects are orthogonal to the linear and cubic time trend components. The standard run order of all other factorial designs considered in this paper exhibits a similar trend robustness pattern to the one shown in Table 6 .

Run orders 1 and 2 in Table 5 perform well in terms of trend robustness. Tables 7 and 8 


\begin{tabular}{|c|c|c|c|c|c|c|}
\cline { 3 - 7 } \multicolumn{1}{c|}{} & \multicolumn{3}{c|}{ Second-order effects } \\
\cline { 2 - 7 } \multicolumn{1}{c|}{ ME } & \multicolumn{2}{c|}{ IE } & \multicolumn{2}{c|}{ QE } \\
\cline { 2 - 7 } & ave & max & ave & max & ave & max \\
\hline L & 0.454 & 0.943 & 0 & 0 & 0 & 0 \\
\hline Q & 0 & 0 & 0.320 & 0.665 & 0.152 & 0.407 \\
\hline C & 0.248 & 0.426 & 0 & 0 & 0 & 0 \\
\hline
\end{tabular}

Table 6: Absolute correlations of the standard run order for the $3^{3}$ design

\begin{tabular}{|c|c|c|c|c|c|c|}
\cline { 3 - 7 } \multicolumn{2}{c|}{} & \multicolumn{3}{c|}{ Second-order effects } \\
\cline { 2 - 7 } \multicolumn{1}{c|}{} & \multicolumn{2}{c|}{ ME } & \multicolumn{2}{c|}{ IE } & \multicolumn{2}{c|}{ QE } \\
\hline & ave & max & ave & max & ave & max \\
\hline L & 0 & 0 & 0.307 & 0.563 & 0.105 & 0.186 \\
\hline Q & 0 & 0 & 0.219 & 0.527 & 0.100 & 0.208 \\
\hline C & $2.22 \cdot 10^{-3}$ & $5.91 \cdot 10^{-3}$ & 0.213 & 0.458 & 0.091 & 0.112 \\
\hline
\end{tabular}

Table 7: Absolute correlations of run order 1 for the $3^{3}$ design

present their trend robustness results. When using run order 1, the main effects are orthogonal to the linear and quadratic time trend components and almost orthogonal to the cubic one. The average absolute correlation of $2.22 \cdot 10^{-3}$ corresponds to an angle that deviates only $0.13^{\circ}$ from orthogonality. The maximum deviation is $0.34^{\circ}$. The other effects are orthogonal to none of the three components of the time trend when using run order 1 . Unlike run order 1, run order 2 in Table 5 does take interaction effects and quadratic effects into account. All effects are orthogonal to the linear time trend component and the main effects are also orthogonal to the quadratic time trend component. Remarkably, the interaction effects and the quadratic effects are also orthogonal to the cubic time trend component.

Figure 2 shows the trend robustness results for the $3^{3}$ factorial design using a heatmap. The heatmaps show, for each model effect and each time trend component, the absolute correlation. To create these figures, we have used a 5-color scale with cut-off points at 0.01 , 0.1, 0.25, 0.5 and 1. This scale is shown in Figure 1. The more the trend robustness degrades, the darker the various cells of the heatmap. Thus, white indicates perfect trend robustness and black indicates the lack of it.

\begin{tabular}{|c|c|c|c|c|c|c|}
\cline { 3 - 7 } \multicolumn{1}{c|}{} & \multicolumn{3}{c|}{ Second-order effects } \\
\cline { 2 - 7 } \multicolumn{1}{c|}{} & \multicolumn{2}{c|}{ ME } & \multicolumn{2}{c|}{ IE } & \multicolumn{2}{c|}{ QE } \\
\cline { 2 - 7 } & ave & max & ave & max & ave & max \\
\hline L & 0 & 0 & 0 & 0 & 0 & 0 \\
\hline Q & 0 & 0 & 0.176 & 0.283 & 0.057 & 0.090 \\
\hline C & 0.318 & 0.727 & 0 & 0 & 0 & 0 \\
\hline
\end{tabular}

Table 8: Absolute correlations of run order 2 for the $3^{3}$ design 


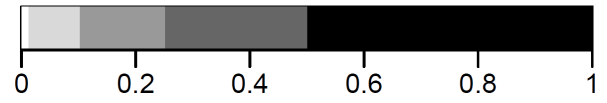

Figure 1: Color coding of the heatmaps

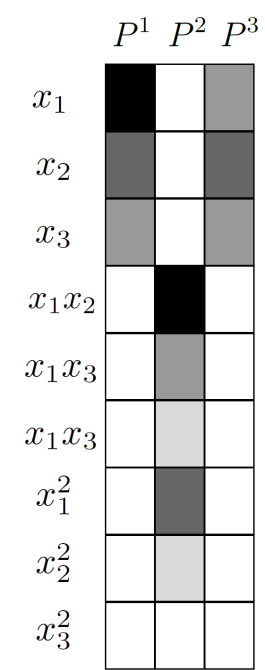

Standard run order

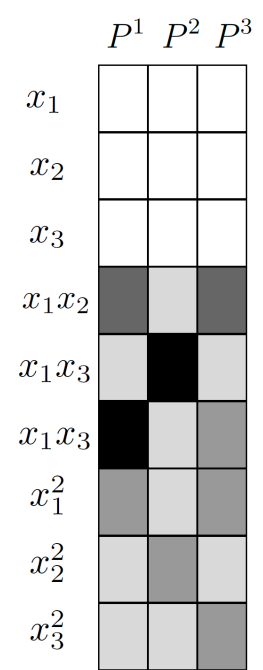

Run order 1

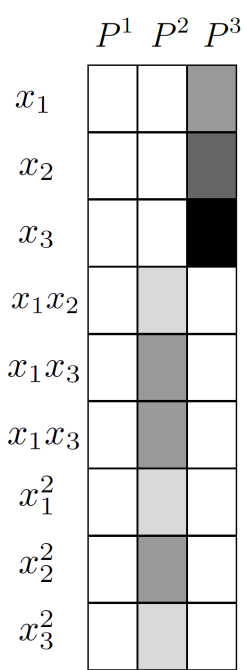

Run order 2

Figure 2: Heatmaps for the $3^{3}$ factorial design

\subsubsection{Summary}

The multistage approach was used for all factorial designs considered. For the small designs, the $3^{2}$ and $4^{2}$ factorial designs, the optimization problems were solved to optimality. For the rest of the designs, the optimality of our results could not be established within the computing time limit. Therefore, the results for the other designs may be subject to improvement.

Tables 9 and 10 summarize our results for the factorial designs we studied, for a main effects model and a full quadratic model, respectively. We consider a model effect to be orthogonal to a time trend if their absolute correlation is smaller than 0.01 . We have obtained run orders for which all main effects are orthogonal to linear and quadratic time trend components for all designs except for the $5^{4}$ factorial design. For the $3^{3}, 3^{4}, 3^{5}, 4^{2}, 4^{3}, 5^{2}$ and $5^{3}$ designs, we also achieved orthogonality to the cubic time trend component. When considering a full quadratic model, we found run orders for the $3^{2}, 3^{3}, 3^{4}, 4^{2}, 4^{3}, 4^{4}, 5^{2}$ and $5^{3}$ factorial designs which are fully orthogonal to the linear time trend component and for which, additionally, the main effects are orthogonal to quadratic time trend component.

These results differ from those of John (1990), as we obtained designs which are robust against linear, quadratic and cubic time trend components, treated the factors as quantitative and used a response surface model. John (1990) only considered linear and quadratic 


\begin{tabular}{|c|c|c|c|c|c|c|c|c|c|c|}
\hline & $3^{2}$ & $3^{3}$ & $3^{4}$ & $3^{5}$ & $4^{2}$ & $4^{3}$ & $4^{4}$ & $5^{2}$ & $5^{3}$ & $5^{4}$ \\
\hline $\mathbf{L}$ & $\checkmark$ & $\sqrt{ }$ & $\checkmark$ & $\checkmark$ & $\checkmark$ & $\checkmark$ & $\checkmark$ & $\checkmark$ & $\checkmark$ & $\checkmark$ \\
\hline $\mathbf{Q}$ & $\checkmark$ & $\sqrt{ }$ & $\checkmark$ & $\sqrt{ }$ & $\checkmark$ & $\checkmark$ & $\checkmark$ & $\checkmark$ & $\checkmark$ & $x$ \\
\hline $\mathbf{C}$ & $x$ & $\checkmark$ & $\checkmark$ & $\checkmark$ & $\checkmark$ & $\checkmark$ & $x$ & $\checkmark$ & $x$ & $\checkmark$ \\
\hline
\end{tabular}

Table 9: Trend robustness of run orders for factorial designs for a main effects model against a linear (L), quadratic $(\mathrm{Q})$ and cubic $(\mathrm{C})$ time trend

\begin{tabular}{|c|c|c|c|c|c|c|c|c|c|c|c|}
\cline { 2 - 11 } \multicolumn{2}{c|}{} & $3^{2}$ & $3^{3}$ & $3^{4}$ & $3^{5}$ & $4^{2}$ & $4^{3}$ & $4^{4}$ & $5^{2}$ & $5^{3}$ & $5^{4}$ \\
\hline \multirow{2}{*}{$\mathrm{L}$} & ME & $\checkmark$ & $\checkmark$ & $\checkmark$ & $\checkmark$ & $\checkmark$ & $\checkmark$ & $\checkmark$ & $\checkmark$ & $\checkmark$ & $\checkmark$ \\
\cline { 2 - 12 } & SOE & $\checkmark$ & $\checkmark$ & $\checkmark$ & $\checkmark$ & $\checkmark$ & $\checkmark$ & $\checkmark$ & $\checkmark$ & $\checkmark$ & $x$ \\
\hline \multirow{2}{*}{$\mathrm{Q}$} & ME & $\checkmark$ & $\checkmark$ & $\checkmark$ & $x$ & $\checkmark$ & $\checkmark$ & $\checkmark$ & $\checkmark$ & $\checkmark$ & $x$ \\
\cline { 2 - 12 } & SOE & $x$ & $x$ & $x$ & $x$ & $x$ & $x$ & $x$ & $\checkmark$ & $\checkmark$ & $x$ \\
\hline \multirow{2}{*}{ C } & ME & $x$ & $x$ & $x$ & $x$ & $\checkmark$ & $x$ & $x$ & $x$ & $x$ & $x$ \\
\cline { 2 - 12 } & SOE & $\checkmark$ & $\checkmark$ & $x$ & $x$ & $\checkmark$ & $x$ & $x$ & $x$ & $x$ & $x$ \\
\hline
\end{tabular}

Table 10: Trend robustness of run orders for factorial designs for a full quadratic model, with main effects $(\mathrm{ME})$ and second-order effects (SOE)

time trends and assumed all factors were categorical. In a similar way, our results for the $3^{2}$ and $4^{2}$ factorial designs also differ from those of Bailey et al. (1992).

Comparing Tables 9 and 10, we can see that considering second-order effects implies that main effects are generally no longer orthogonal to the cubic time trend component.

\subsection{Results for response surface designs with 3 factors}

\subsubsection{Central composite designs (CCD)}

For the 3-factor face-centered CCD with one center point, we found two attractive run orders which we characterize in Table 11. Tables 12 and 13 show the absolute correlations between the model effects and the time trend components of these run orders. For this CCD, our multistage optimization was solved to optimality, so these run orders are the best ones possible.

On the one hand, run order 1 ensures that the main effects are orthogonal to the linear and quadratic time trend components but not to the cubic one. On the other hand, run order 2 achieves orthogonality between the main effects and interaction effects, and the linear time trend component. The quadratic model effects are nearly orthogonal to the linear time trend component, since the maximum deviation from orthogonality equals $90^{\circ}-\cos ^{-1}(0.038)=2.18^{\circ}$. Similarly, the main effects are nearly orthogonal to the quadratic time trend component. 


\begin{tabular}{|c|r|r|r|r|r|r|r|r|r|}
\cline { 2 - 11 } \multicolumn{1}{c|}{} & \multicolumn{3}{c|}{ Run order 1 } & \multicolumn{3}{c|}{ Run order 2 } & \multicolumn{3}{c|}{ Time trend } \\
\hline $\mathbf{t}$ & $X_{1 t}$ & $X_{2 t}$ & $X_{3 t}$ & $X_{1 t}$ & $X_{2 t}$ & $X_{3 t}$ & $Z_{1 t}$ & $Z_{2 t}$ & $Z_{3 t}$ \\
\hline $\mathbf{1}$ & 0 & 1 & 0 & 0 & -1 & 0 & -7 & 91 & -91 \\
\hline $\mathbf{2}$ & 0 & 0 & -1 & 1 & 1 & -1 & -6 & 52 & -13 \\
\hline $\mathbf{3}$ & 1 & -1 & 1 & -1 & 1 & 1 & -5 & 19 & 35 \\
\hline $\mathbf{4}$ & -1 & -1 & -1 & 0 & 0 & 1 & -4 & -8 & 58 \\
\hline $\mathbf{5}$ & -1 & 1 & 1 & -1 & -1 & -1 & -3 & -29 & 61 \\
\hline $\mathbf{6}$ & 0 & 0 & 1 & 1 & 0 & 0 & -2 & -44 & 49 \\
\hline $\mathbf{7}$ & 1 & -1 & -1 & 1 & -1 & 1 & -1 & -53 & 27 \\
\hline $\mathbf{8}$ & 1 & 1 & 1 & -1 & 0 & 0 & 0 & -56 & 0 \\
\hline $\mathbf{9}$ & 0 & -1 & 0 & -1 & 1 & -1 & 1 & -53 & -27 \\
\hline $\mathbf{1 0}$ & -1 & 0 & 0 & 0 & 0 & -1 & 2 & -44 & -49 \\
\hline $\mathbf{1 1}$ & -1 & 1 & -1 & 1 & 1 & 1 & 3 & -29 & -61 \\
\hline $\mathbf{1 2}$ & 0 & 0 & 0 & 0 & 0 & 0 & 4 & -8 & -58 \\
\hline $\mathbf{1 3}$ & 1 & 1 & -1 & 1 & -1 & -1 & 5 & 19 & -35 \\
\hline $\mathbf{1 4}$ & 1 & 0 & 0 & -1 & -1 & 1 & 6 & 52 & 13 \\
\hline $\mathbf{1 5}$ & -1 & -1 & 1 & 0 & 1 & 0 & 7 & 91 & 91 \\
\hline
\end{tabular}

Table 11: Trend-robust run orders of a 3-factor face-centered CCD with one center point with coded time trend

\begin{tabular}{|c|c|c|c|c|c|c|}
\cline { 3 - 7 } \multicolumn{2}{c|}{} & \multicolumn{3}{c|}{ Second-order effects } \\
\cline { 2 - 7 } \multicolumn{1}{c|}{} & \multicolumn{2}{c|}{ ME } & \multicolumn{2}{c|}{ IE } & \multicolumn{2}{c|}{ QE } \\
\cline { 2 - 7 } \multicolumn{1}{c|}{} & ave & max & ave & max & ave & max \\
\hline L & 0 & 0 & 0.324 & 0.380 & 0.126 & 0.189 \\
\hline Q & 0 & 0 & 0.297 & 0.451 & 0.046 & 0.062 \\
\hline C & 0.333 & 0.492 & 0.165 & 0.206 & 0.217 & 0.336 \\
\hline
\end{tabular}

Table 12: Absolute correlations of run order 1 for the 3-factor face-centered CCD with one center point

In this case, adding a couple of center runs is beneficial to obtain better orthogonality results, as it enables us to construct a run order for which all model effects are orthogonal to a linear time trend and all main effects are orthogonal to a quadratic time trend component. The absolute correlations between the model effects and the time trend components for an extended 3-factor face-centered CCD with three center runs are given in Table 14 .

\begin{tabular}{|c|c|c|c|c|c|c|}
\cline { 2 - 7 } \multicolumn{1}{c|}{} & \multicolumn{3}{c|}{ Second-order effects } \\
\cline { 2 - 7 } \multicolumn{1}{c|}{ ME } & \multicolumn{2}{c|}{ IE } & \multicolumn{2}{c|}{ QE } \\
\cline { 2 - 7 } \multicolumn{1}{c|}{ ave } & max & ave & max & ave & max \\
\hline L & 0 & 0 & 0 & 0 & 0.025 & 0.038 \\
\hline C & 0.026 & 0.059 & 0.268 & 0.561 & 0.195 & 0.263 \\
\hline
\end{tabular}

Table 13: Absolute correlations of run order 2 for the 3 -factor face-centered CCD with one center point 


\begin{tabular}{|c|c|c|c|c|c|c|}
\cline { 3 - 7 } \multicolumn{1}{c|}{} & \multicolumn{3}{c|}{ Second-order effects } \\
\cline { 2 - 7 } \multicolumn{1}{c|}{} & \multicolumn{2}{c|}{ ME } & \multicolumn{2}{c|}{ IE } & \multicolumn{2}{c|}{ QE } \\
\cline { 2 - 7 } & ave & max & ave & max & ave & max \\
\hline L & 0 & 0 & 0 & 0 & 0 & 0 \\
\hline Q & 0 & 0 & 0.270 & 0.490 & 0.407 & 0.503 \\
\hline C & 0.430 & 0.762 & 0 & 0 & 0 & 0 \\
\hline
\end{tabular}

Table 14: Absolute correlations of the best run order found for the 3-factor face-centered CCD with three center points

\begin{tabular}{|c|c|c|c|c|c|c|}
\cline { 3 - 7 } \multicolumn{2}{c|}{} & \multicolumn{3}{c|}{ Second-order effects } \\
\cline { 2 - 7 } \multicolumn{1}{c|}{} & \multicolumn{2}{c|}{ ME } & \multicolumn{2}{c|}{ IE } & \multicolumn{2}{c|}{ QE } \\
\cline { 2 - 7 } \multicolumn{1}{c|}{} & ave & max & ave & max & ave & max \\
\hline L & 0 & 0 & 0.175 & 0.334 & 0.208 & 0.389 \\
\hline Q & 0.093 & 0.223 & 0.211 & 0.393 & 0.167 & 0.334 \\
\hline C & 0.260 & 0.499 & 0.214 & 0.336 & 0.172 & 0.324 \\
\hline
\end{tabular}

Table 15: Absolute correlations of the best run order found for the 3-factor sCCD

\subsubsection{Small central composite designs (sCCD)}

Table 15 presents the absolute correlations between model effects and time trend components of the trend-robust run order found for a 3-factor sCCD with one center point. The trend robustness improves slightly when we add more center runs. We need seven center points to obtain a run order for which the main effects are orthogonal to linear and quadratic time trend components.

\subsubsection{Box-Behnken designs (BBD)}

For the 3-factor BBD, the six optimization problems in our multistage approach were solved to optimality. The run order in Table 16 is therefore the best possible. The absolute correlations between the model effects and the time trend components are given in Table 17 . The run order we found allows a full quadratic model to be estimated independently from the linear time trend component, while, additionally, the main effects are orthogonal to the quadratic time trend. For 3-factor BBDs, we did not observe better orthogonality results when we added more center runs.

\subsubsection{Definitive screening designs (DSD)}

Table 18 presents the properties of the best run order found for the plain definitive screening design with three factors. Clearly, the best run order is not very trend-robust. 


\begin{tabular}{|c|r|r|r|r|r|r|r|r|r|r|r|r|r|}
\hline $\mathbf{t}$ & \multicolumn{1}{|c|}{$\mathbf{2}$} & \multicolumn{1}{|c|}{$\mathbf{3}$} & \multicolumn{1}{|c|}{} & \multicolumn{1}{|c|}{} & \multicolumn{1}{c}{$\mathbf{6}$} & $\mathbf{7}$ & $\mathbf{8}$ & $\mathbf{9}$ & $\mathbf{1 0}$ & $\mathbf{1 1}$ & $\mathbf{1 2}$ & $\mathbf{1 3}$ \\
\hline$X_{1 t}$ & 1 & -1 & -1 & 1 & 0 & 0 & 0 & 0 & 0 & -1 & 1 & 1 & -1 \\
\hline$X_{2 t}$ & 0 & -1 & 1 & 0 & 1 & -1 & 0 & 1 & -1 & 0 & -1 & 1 & 0 \\
\hline$X_{3 t}$ & -1 & 0 & 0 & 1 & 1 & 1 & 0 & -1 & -1 & -1 & 0 & 0 & 1 \\
\hline$Z_{1 t}$ & -6 & -5 & -4 & -3 & -2 & -1 & 0 & 1 & 2 & 3 & 4 & 5 & 6 \\
\hline$Z_{2 t}$ & 22 & 11 & 2 & -5 & -10 & -13 & -14 & -13 & -10 & -5 & 2 & 11 & 22 \\
\hline$Z_{3 t}$ & -11 & 0 & 6 & 8 & 7 & 4 & 0 & -4 & -7 & -8 & -6 & 0 & 11 \\
\hline
\end{tabular}

Table 16: Trend-robust run order of a 3-factor Box-Behnken design with coded time trend

\begin{tabular}{|c|c|c|c|c|c|c|}
\cline { 3 - 7 } \multicolumn{2}{c|}{} & \multicolumn{3}{c|}{ Second-order effects } \\
\cline { 2 - 7 } \multicolumn{1}{c|}{} & \multicolumn{2}{c|}{ ME } & \multicolumn{2}{c|}{ IE } & \multicolumn{2}{c|}{ QE } \\
\cline { 2 - 7 } \multicolumn{1}{c|}{} & ave & max & ave & max & ave & max \\
\hline L & 0 & 0 & 0 & 0 & 0 & 0 \\
\hline Q & 0 & 0 & 0.291 & 0.603 & 0.242 & 0.474 \\
\hline C & 0.473 & 0.887 & 0 & 0 & 0 & 0 \\
\hline
\end{tabular}

Table 17: Absolute correlations of the best run order found for for the 3-factor Box-Behnken design

As explained in Section 2.1, we can extend a definitive screening design by selecting a subset of columns from a design with a larger number of factors. We obtained good trendrobust results for a 3-factor design based on 8-factor DSD. The best run orders found are shown in Table 19. Tables 20 (main effects model) and 21 (full quadratic model) show the absolute correlations of these run orders. Unlike the original 3-factor design, this 17-run design does allow trend-robust run orders to be constructed, with properties similar to those of the 3-factor CCD.

\subsection{Summary of results for response surface designs}

We identified run orders for response surface designs with three up to six factors with trend robustness properties similar to those exposed in Tables 20 and 21. In other words, we have found run orders for which the main effects are orthogonal to the linear and quadratic time trend components, and for which, additionally, the second-order effects are orthogonal to the linear time trend component. To summarize our results graphically, we have used the

\begin{tabular}{|c|c|c|c|c|c|c|}
\cline { 3 - 7 } \multicolumn{2}{c|}{} & \multicolumn{3}{c|}{ Second-order effects } \\
\cline { 2 - 7 } \multicolumn{1}{c|}{} & \multicolumn{2}{c|}{ ME } & \multicolumn{2}{c|}{ IE } & \multicolumn{2}{c|}{ QE } \\
\cline { 2 - 7 } \multicolumn{1}{c|}{} & ave & max & ave & max & ave & max \\
\hline L & 0.053 & 0.053 & 0.516 & 0.775 & 0.158 & 0.264 \\
\hline Q & 0.116 & 0.302 & 0.304 & 0.342 & 0.194 & 0.489 \\
\hline C & 0.285 & 0.402 & 0.350 & 0.667 & 0.208 & 0.467 \\
\hline
\end{tabular}

Table 18: Absolute correlations of the best run order found for the 3-factor definitive screening design 


\begin{tabular}{|c|r|r|r|r|r|r|r|r|r|}
\cline { 2 - 11 } \multicolumn{1}{c}{} & \multicolumn{3}{c|}{ Run order 1 } & \multicolumn{3}{c|}{ Run order 2 } & \multicolumn{3}{c|}{ Time trend } \\
\hline $\mathbf{t}$ & $X_{1 t}$ & $X_{2 t}$ & $X_{3 t}$ & $X_{1 t}$ & $X_{2 t}$ & $X_{3 t}$ & $Z_{1 t}$ & $Z_{2 t}$ & $Z_{3 t}$ \\
\hline $\mathbf{1}$ & 1 & -1 & 0 & -1 & 0 & -1 & -8 & 40 & -28 \\
\hline $\mathbf{2}$ & -1 & 1 & 0 & -1 & -1 & 1 & -7 & 25 & -7 \\
\hline $\mathbf{3}$ & 1 & 1 & -1 & 0 & 1 & 1 & -6 & 12 & 7 \\
\hline $\mathbf{4}$ & -1 & -1 & 1 & 1 & 1 & -1 & -5 & 1 & 15 \\
\hline $\mathbf{5}$ & -1 & -1 & 1 & 1 & -1 & -1 & -4 & -8 & 18 \\
\hline $\mathbf{6}$ & -1 & 1 & -1 & 1 & 1 & 1 & -3 & -15 & 17 \\
\hline $\mathbf{7}$ & 1 & 1 & 1 & 1 & -1 & 0 & -2 & -20 & 13 \\
\hline $\mathbf{8}$ & 0 & -1 & -1 & 1 & -1 & 1 & -1 & -23 & 7 \\
\hline $\mathbf{9}$ & 1 & 1 & -1 & 0 & 0 & 0 & 0 & -24 & 0 \\
\hline $\mathbf{1 0}$ & 1 & 0 & 1 & -1 & 1 & -1 & 1 & -23 & -7 \\
\hline $\mathbf{1 1}$ & 1 & -1 & 1 & -1 & 1 & 0 & 2 & -20 & -13 \\
\hline $\mathbf{1 2}$ & -1 & -1 & -1 & -1 & -1 & -1 & 3 & -15 & -17 \\
\hline $\mathbf{1 3}$ & -1 & 0 & -1 & -1 & 1 & 1 & 4 & -8 & -18 \\
\hline $\mathbf{1 4}$ & 0 & 0 & 0 & -1 & -1 & 1 & 5 & 1 & -15 \\
\hline $\mathbf{1 5}$ & 0 & 1 & 1 & 0 & -1 & -1 & 6 & 12 & -7 \\
\hline $\mathbf{1 6}$ & -1 & 1 & 1 & 1 & 1 & -1 & 7 & 25 & 7 \\
\hline $\mathbf{1 7}$ & 1 & -1 & -1 & 1 & 0 & 1 & 8 & 40 & 28 \\
\hline
\end{tabular}

Table 19: Trend-robust run orders of a 17-run 3-factor DSD with coded time trend

\begin{tabular}{|c|c|c|c|c|c|c|}
\cline { 2 - 7 } \multicolumn{2}{c|}{} & \multicolumn{3}{c|}{ Second-order effects } \\
\cline { 2 - 7 } \multicolumn{1}{c|}{} & \multicolumn{2}{c|}{ ME } & \multicolumn{2}{c|}{ IE } & \multicolumn{2}{c|}{ QE } \\
\cline { 2 - 7 } \multicolumn{1}{c|}{} & ave & max & ave & max & ave & max \\
\hline L & 0 & 0 & 0.262 & 0.529 & 0.132 & 0.132 \\
\hline C & 0 & 0 & 0.403 & 0.489 & 0.107 & 0.200 \\
\hline
\end{tabular}

Table 20: Absolute correlations of run order 1 for the 17-run 3-factor design based on a 8-factor definitive screening design

following representation for the various designs:

3 central composite design with three center points

$\lceil$ 1」: small central composite design with one center point

1.: Box-Behnken design with one center point

11. design based on a definitive screening design for 11 factors

Figure 3 shows, for any given number of factors and type of design, the smallest design with a run order for which the main effects are robust against linear and quadratic time trend components. 


\begin{tabular}{|c|c|c|c|c|c|c|}
\cline { 3 - 7 } \multicolumn{2}{c|}{} & \multicolumn{3}{c|}{ Second-order effects } \\
\cline { 2 - 7 } \multicolumn{1}{c|}{} & \multicolumn{2}{c|}{ ME } & \multicolumn{2}{c|}{ IE } & \multicolumn{2}{c|}{ QE } \\
\cline { 2 - 7 } \multicolumn{1}{c|}{} & ave & max & ave & max & ave & max \\
\hline L & 0 & 0 & 0 & 0 & 0 & 0 \\
\hline Q & 0 & 0 & 0.201 & 0.407 & 0.121 & 0.194 \\
\hline C & 0.378 & 0.901 & 0 & 0 & 0 & 0 \\
\hline
\end{tabular}

Table 21: Absolute correlations of run order 2 for the 17-run 3-factor design based on a 8-factor definitive screening design

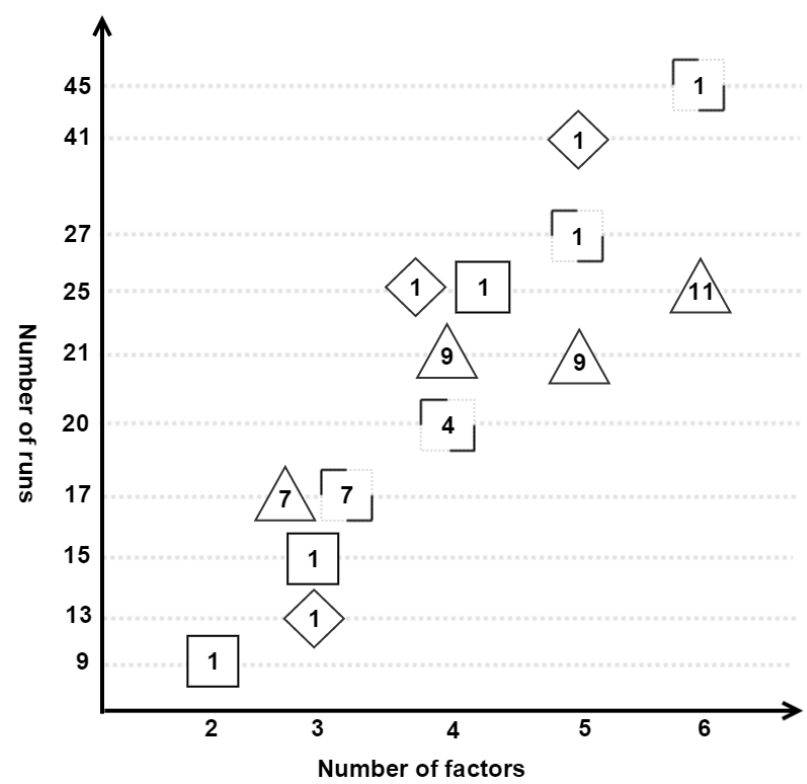

Figure 3: Response surface designs with a run order for which the main effects are orthogonal to linear and quadratic time trend components

For the scenario with 2 factors, we have found a run order for a CCD with one center point for which the main effects are orthogonal to the linear and quadratic time trend components. For none of the 2-factor sCCDs we considered, we obtained attractive trend-robust run orders. For 3 and 4 factors, we did find trend-robust run orders for all types of response surface designs. Here, it was often necessary to extend the design to obtain trend-robust run orders. For example, a trend-robust run order exists for the 3-factor design based on a 8-factor DSD (which has 17 runs and which is shown in Table 19). We found a trend-robust run order for the 4 -factor sCCD with 4 center points. For five and six factors, the definitive screening designs for which trend-robust run orders exist are significantly smaller than the trend-robust small central composite designs.

Figure 4 shows, for a given number of factors and type of design, the smallest design for which a run order exists so that the main effects are robust against the linear and quadratic time trend components and the second-order effects are orthogonal to the linear time trend 


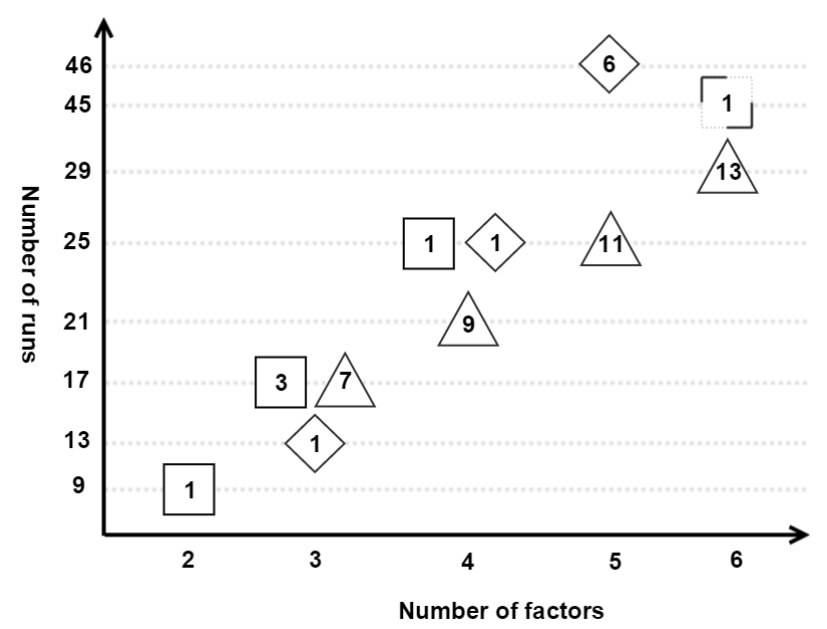

Figure 4: Response surface designs with a run order for which the main effects are orthogonal to linear and quadratic time trend components and the second-order effects are orthogonal to linear time trend component

component.

Interestingly, Figure 4 shows fewer designs than Figure 3. This indicates that it is harder to obtain good trend robustness results when a full quadratic model is considered. For example, the multistage optimization indicated that no trend-robust run orders exist for the 2-factor, 3-factor and 4-factor sCCDs. Again, we observe that extending the designs is often beneficial for achieving trend-robustness. For example, the 4-factor design based on a 10factor DSD (21 runs) has a trend-robust run order for which the main effects are orthogonal to linear and quadratic time trends components and for which the second-order effects are orthogonal to the linear time trend component. Analogously, the 5-factor design based on a 12-factor DSD (25 runs) meets these orthogonality conditions. The 5- and 6-factor DSD have good trend-robustness properties compared to the other response surface designs despite their small size. Finally, we found robust run orders for 3 -factor, 4 -factor and 5 -factor Box-Behnken designs.

Detailed results for the robustness of the designs studied are included in the appendix.

\section{Discussion and extensions}

Our multistage approach is an effective way of re-ordering the runs of a given response surface design to achieve robustness against polynomial time trends. We improved the results of some existing papers on this topic, provided a general method which can be applied to an arbitrary design and we showed how extending the design is sometimes beneficial for the trend robustness. Some future research possibilities naturally arise. First, we could inves- 
tigate whether axial points for central composite designs can be found such that we can obtain a better trend robustness than for the face-centered central composite designs. A second topic could be the application of our multistage algorithm to other designs, such as mixture designs or multi-level and mixed-level (fractional) factorial designs, while treating the factors as qualitative rather than quantitative.

With minor modifications, the approach we propose here can deal with different scenarios as well. The first scenario or related problem occurs when the number of available time slots to conduct the experiments is larger than the number of runs of the design considered. Some time slots will then be idle, in the sense that no experimental test will take place. This can be done using a variation of the mixed integer program presented in Section 4.2, where constraints (14) are substituted by

$$
\sum_{j=1}^{n} \pi_{i j} \leq 1, \quad \forall i
$$

so we allow some time slots not to be chosen. In the second scenario, $n$ non-consecutive time slots are available to conduct the $n$ runs of the experimental design. This happens, for example, when the experiments are planned on different days, or at different non-consecutive hours of the same day. The orthogonal polynomials presented in Section 3.1 are then not longer valid. Following the procedure presented in Robson (1959), it is, however, possible to construct alternative orthogonal polynomials to code the time trend components and to find the best run orders using our multistage approach. These two extensions are also considered in Atkinson and Donev (1996). Finally, our approach can easily deal with non polynomial time trends (e.g. exponential) just by using an appropriate model matrix for the time trend.

\section{Supplementary Materials}

The supplementary materials include detailed results for the 30 designs considered in this paper. Additionally, the materials contain txt files with the different run orders that we have found and the orthogonal polynomial time trend components for different numbers of runs. The supplementary materials are available at http://www.asq.org/pub/jqt/. 


\section{References}

Atkinson, A. C. and Donev, A. N. (1996). Experimental designs optimally balanced for trend. Technometrics, 38(4):333-341.

Bailey, R. A., Cheng, C. S., and Kipnis, P. (1992). Construction of trend-resistant factorial designs. Statistica Sinica, 2(2):393-411.

Box, G. E. P. and Behnken, D. W. (1960). Some new three level designs for the study of quantitative variables. Technometrics, 2(4):455-475.

Box, G. E. P. and Hay, W. A. (1953). A statistical design for the efficient removal of trends occurring in a comparative experiment with an application in biological assay. Biometrics, 9(3):304-319.

Box, G. E. P. and Wilson, K. B. (1951). On the experimental attainment of optimum conditions. Journal of the Royal Statistical Society. Series B (Methodological), 13(1):1-45.

Capehart, S. R., Keha, A., Kulahci, M., and Montgomery, D. C. (2011). Designing fractional factorial split-plot experiments using integer programming. International Journal of Experimental Design and Process Optimisation, 2:34-57.

Capehart, S. R., Keha, A., Kulahci, M., and Montgomery, D. C. (2012). Generating blocked fractional factorial split-plot designs using integer programming. International Journal of Experimental Design and Process Optimisation, 3(2):111-132.

Carrano, A. L., Thorn, B. K., and Lopez, G. (2006). An integer programming approach to the construction of trend-free experimental plans on split-plot designs. Journal of Manufacturing Systems, 25(1):39 - 44.

Cheng, C. S. and Jacroux, M. (1988). The construction of trend-free run orders of two-level factorial designs. Journal of the American Statistical Association, 83(404):1152-1158.

Cook, S. A. (1971). The complexity of theorem-proving procedures. In Proceedings of the Third Annual ACM Symposium on Theory of Computing, STOC '71, pages 151-158, New York, NY, USA. ACM.

Cox, D. R. (1951). Some systematic experimental designs. Biometrika, 38(3/4):312-323.

Daniel, D. and Wilcoxon, F. (1966). Factorial $2^{p-q}$ plans robust against linear and quadratic trends. Technometrics, 8(2):259-278. 
Dhanasekaran, S. and Karunanithi, T. (2012). Statistical analysis of experimental variables on gas holdup in novel bubble column using Box-Behnken design. International Journal of Chemical Reactor Engineering, 10(1):1-28.

Fisher, R. A. and Yates, F. (1963). Statistical tables for biological, agricultural and medical research. Oliver and Boyd, London, 6th edition.

Garey, M. R. and Johnson, D. S. (1979). Computers and Intractability: A Guide to the Theory of NP-Completeness. W. H. Freeman \& Co., New York, NY, USA.

Hartley, H. O. (1959). Smallest composite designs for quadratic response surfaces. Biometrics, 15(4):611-624.

Hinkelmann, K. and Jo, J. (1998). Linear trend-free Box-Behnken designs. Journal of Statistical Planning and Inference, 72(1):347-354.

John, P. W. M. (1990). Time trends and factorial experiments. Technometrics, 32(3):275282.

Joiner, B. L. and Campbell, C. (1976). Designing experiments when run order is important. Technometrics, 18(3):249-259.

Jones, B. and Nachtsheim, C. J. (2011). A class of three-level designs for definitive screening in the presence of second-order effects. Journal of Quality Technology, 43(1):1-14.

Kreutz, C. and Timmer, J. (2009). Systems biology: experimental design. FEBS Journal, 276(4):923-942.

Robson, D. S. (1959). A simple method for constructing orthogonal polynomials when the independent variable is unequally spaced. Biometrics, 15(2):187-191.

Sartono, B., Goos, P., and Schoen, E. (2015a). Constructing general orthogonal fractional factorial split-plot designs. Technometrics, 57(4):488-502.

Sartono, B., Schoen, E., and Goos, P. (2015b). Blocking orthogonal designs with mixed integer linear programming. Technometrics, 57(3):428-439.

Tack, L. and Vandebroek, M. (2001). ( $\left.D_{t}, C\right)$-optimal run orders. Journal of Statistical Planning and Inference, 98(12):293 - 310.

Tack, L. and Vandebroek, M. (2002). Trend-resistant and cost-efficient block designs with fixed or random block effects. Journal of Quality Technology, 34(4):422-436. 
Tan, P., Steinbach, M., and Kumar, V. (2014). Introduction to Data Mining. Pearson Education, Limited.

Van der Reyden, D. (1957). The Use of Orthogonal Polynomial Contrasts in the Confounding of Factorial Experiments. PhD thesis, North Carolina State College.

Villarreal-Marroqun, M. G., Chen, P., Mulyana, R., Santner, T. J., Dean, A. M., and Castro, J. M. (2017). Multiobjective optimization of injection molding using a calibrated predictor based on physical and simulated data. Polymer Engineering \& Science, 57(3):248-257. 